\title{
Breast cancer and Flammer syndrome: any symptoms in common for prediction, prevention and personalised medical approach?
}

\author{
Pavol Zubor ${ }^{1,2}$ - Alexandra Gondova ${ }^{1}$. Jiri Polivka $\mathrm{Jr}^{3,4} \cdot$ Petra Kasajova $^{1}$. \\ Katarzyna Konieczka ${ }^{5}$ - Jan Danko ${ }^{1}$ • Olga Golubnitschaja ${ }^{6,7,8}$
}

Received: 7 March 2017 / Accepted: 14 March 2017 /Published online: 10 April 2017

(C) European Association for Predictive, Preventive and Personalised Medicine (EPMA) 2017

\begin{abstract}
An epidemic scale of the breast cancer (BC) prevalence is actually recognised as the reality of the early twentyfirst century. Particularly alarming is that the sporadic BC (about $90 \%$ of all patients) creates currently unpredictable subpopulations in terms of disease predisposition, development and progression. Despite broad discussions run since years in $\mathrm{BC}$ area, no any plausible approach has been suggested so far to get the overall situation better controlled in the populations. Here, we present highly innovative concepts considering investigation of specific syndromes and symptoms underestimated till now in relationship with $\mathrm{BC}$ predisposition and development. Consequently, the purpose of our pilot project was to evaluate the prevalence of Flammer Syndrome
\end{abstract}

Olga Golubnitschaja

olga.golubnitschaja@ukbonn.de

1 Department of Obstetrics and Gynaecology, Jessenius Faculty of Medicine, Martin University Hospital, Martin, Slovak Republic

2 Division of Oncology, Biomedical Centre Martin, Jessenius Faculty of Medicine, Comenius University in Bratislava, Bratislava, Slovak Republic

3 Department of Histology and Embryology and Biomedical Center, Faculty of Medicine in Plzen, Charles University, Prague, Czech Republic

4 Department of Neurology, Faculty Hospital Plzen, Prague, Czech Republic

5 Department of Ophthalmology, University of Basel, Basel, Switzerland

6 Radiological clinic, Rheinische Friedrich-Wilhelms-University of Bonn, Sigmund-Freud-Str 25, 53105 Bonn, Germany

7 Breast Cancer Research Centre, Rheinische Friedrich-Wilhelms-University of Bonn, Bonn, Germany

8 Centre for Integrated Oncology, Cologne-Bonn, Rheinische Friedrich-Wilhelms-University of Bonn, Bonn, Germany
(FS) in BC patient cohort. The results achieved here support the main hypothesis of the project clearly demonstrating the tendency of BC patients to the increased prevalence of FS symptoms compared to the disease-free individuals. Our study strongly indicates the relevance of FS symptoms for BC pathology such as feeling inadequately cold, deficient thermoregulation, altered sensitivity to different stimuli, potential dehydration, altered sleep patterns, tendency towards headache, migraine attacks and dizziness. Moreover, the symptoms' appearance is specifically linked to the individual BC subtypes. Potential mechanisms interconnecting FS with BC pathology are discussed.

Keywords Predictive preventive personalised medicine · Breast cancer · Flammer syndrome - Systemic hypoxia · Patient stratification · Pain - Thermoregulation · Cardiovascular component $\cdot$ Drug sensitivity $\cdot$ Sense regulation

\section{Introduction}

An epidemic scale of the breast cancer (BC) prevalence is actually recognised as the reality of the early twenty-first century [1]. Further, it has been demonstrated that more than $50 \%$ of all BC cases are well preventable, where modifiable risk factors play the major role in the disease predisposition and development [1]. The "familial BC" cases characterised by the inherited genetic component dominating in the disease aetiology, comprise not more than $10 \%$ of the entire $\mathrm{BC}$ patient cohort that represents only the tip of the whole BC "iceberg". In contrast, the main body of this iceberg is represented by so-called "sporadic BC" comprising about $90 \%$ of cases. Particularly alarming is the actuality that specifically sporadic $\mathrm{BC}$ creates currently unpredictable subpopulations in terms of all three factors, namely disease predisposition, development and progression. Despite 
broad discussions run since years in $\mathrm{BC}$ area, no any plausible approach has been suggested so far to get the overall situation better controlled in the populations.

However, on a positive note, potential solutions start to be crystallised at the multiprofessional level now [2], such as the urgent necessity to introduce

- innovative screening programmes focused on the young subpopulations (teenagers + ) obligatory considering an interplay between contributing internal and external risk factors

- investigation of specific syndromes and symptoms underestimated till now in relationship with $\mathrm{BC}$ predisposition, development and progression

- new approaches for more precise/personalised patient phenotyping and stratification

- creation of the pathology-specific individual patient profiles, multilevel diagnostics approaches, novel biomarker patterns

- advanced educational measures for both professionals and general population strongly focused on an effective prevention of sporadic BC.

Actual research article follows the above-listed recommendations contributing specifically to the point "investigation of specific syndromes and symptoms underestimated till now in relationship with BC predisposition and development". The facts and hypotheses which motivate and justify this research work have been recently presented in the review article "Feeling cold and other underestimated symptoms in breast cancer: anecdotes or individual profiles for advanced patient stratification?" [2]. In brief, we summarise here the most issue-relevant considerations. As it has been explained in detail in the above noted review article, individual symptoms listed below have been described in the literature in the context of $\mathrm{BC}$, namely,

- deficient thermoregulation

- feeling inadequately cold

- persistently cold extremities

- altered sensitivity to different stimuli: pain, thirst, smell, light, stress provocation (including internal and external stress factors)

- dehydration

- altered circadian and sleep patterns

- tendency towards headache, migraine attacks and dizziness

- tendency towards abnormal BMI

- altered endothelin-1 blood patterns

- local and systemic hypoxic effects.

However, all these phenomena are strongly underinvestigated and consequently underestimated in the overall $\mathrm{BC}$ management. On the other hand, the above-listed symptoms are characteristic for the Flammer Syndrome (FS) phenotype extensively described in 2014 by the dedicated research group [3]. FS is described for both healthy individuals in suboptimal health condition and severely diseased patients $[4,5]$. Consequently, our particular interest to FS in the context of $\mathrm{BC}$ research and management may be formulated as follows:

- Onset of FS symptoms is observed early in life (puberty)

- FS is more typical for young women

- Systemic molecular events characteristic for FS are evidently involved into effective $\mathrm{BC}$ cancer advancement [5-8]

- Local and systemic hypoxic effects caused by vascular dysregulation may strongly predispose FS-individuals to the formation of "premetastatic niches" described as the "fertile" microenvironment for particularly aggressive $\mathrm{BC}$ subtypes characterised by quickly progressing metastatic disease [1].

The actual article investigates the prevalence of FS in individual subtypes of $\mathrm{BC}$ versus $\mathrm{BC}$-free individuals.

\section{Materials and methods}

\section{Flammer syndrome diagnostic approach}

The Flammer Syndrome (FS) describes the phenotype of people with a predisposition to an altered reaction of the blood vessels towards different stimuli such as cold provocation and emotional stress. Frequent symptoms are the following ones: cold hands and/or feet; low blood pressure; prolonged sleep onset time; reduced feeling of thirst and increased sensitivity to odour, pain, vibration and certain drugs. Frequent signs are altered gene expression, prolonged blood flow cessation monitored by the nailfold-capillaroscopy after the cold provocation and reduced autoregulation of ocular blood flow. FS occurs more often in females than in males, in thin than in obese individuals, in young than in old people, in academics than in blue collar workers and in subjects with indoor than outdoor jobs [3]. FS influences the regulation of retinal venous pressure [9] that is well described for the glaucoma pathology [10]. FS has been functionally linked to the retinal vein occlusion [11]. FS plays a role in the pathology of retinitis pigmentosa [12]. Symptoms of FS have been described in patients suffering from multiple sclerosis [13].

The FS questionnaire applied to the actual study has been developed at the University Hospital Basel, Switzerland. It is based on the knowledge currently available and collected since the phenomenon has been described $[3,14,15]$. The actual version of the FS questionnaire has been successfully applied to study different populations [16], FS symptomatic in 
retinitis pigmentosa [17] as well as in multiple sclerosis [13] and other clinically relevant patient cohorts $[18,19]$. With the permanently growing knowledge about the FS, the dedicated questionnaire should be, further, adapted and improved.

Actually applied questionnaire comprises 15 questions altogether describing the most relevant FS symptoms, namely,

1. Do you suffer from cold hands and/or feet?

2. Do you feel cold when you are sitting quietly for some time or when you are not moving?

3. Do you have (or have earlier had) a low blood pressure?

4. Do you ever feel dizzy when you suddenly stand up from a lying (or resting) position?

5. Do you need a relatively long time to fall asleep?

6. How is your feeling of thirst?

7. How often do you have headache?

8. In case you suffer from migraines, do you have accompanying symptoms (e.g. visual disturbances, transient altered sensation such as cribbling in your arms or in your legs, etc.)?

9. If you have to take medications (other than painkillers), do you have the feeling that you react strongly to them and/or that you would feel better if you would take a lower dose than that which is normally prescribed?

10. Do you suffer from any type of pain which you would have to take pain killers for?

11. How well can you smell: can you smell things that other people do not smell or that others smell to a lesser extent?

12. Were you slim at 20-30 years of age?

13. If you have to judge yourself (e.g. in your work), would you say that you are particularly reliable with a tendency towards perfectionism?

14. Have you had phases in your life in which you had ringing in your ears (tinnitus)?

15. Have you noticed reversible blotches (white or red) on your skin when you were very excited or angry (e.g. in stress)?

\section{Patients' recruitment and examinations}

The patient database available at the Department of Obstetrics and Gynaecology, Jessenius Faculty of Medicine, Martin University Hospital, Martin, Slovak Republic has been utilised for selecting the patients as potential responders for the above noted FS questionnaire. The following diagnoses have been chosen as the including criterion:

- breast cancer (BC) main types stratified as luminal, Her2positive and triple-negative ones

- benign breast fibro-adenoma (FIA), free of breast cancer and any other malignancy
- healthy individuals free of breast cancer and any other malignancy.

Altogether, 155 patients have been recruited for the current study divided into the following groups: 73 breast cancer-free (21 FIA and 52 healthy) and 82 breast cancer-diseased individuals stratified as luminal (51), Her-2 positive (13) and triple-negative (18) BC. All participants have been informed about the purpose of and their rights as participants in the study. All investigations conformed to the ethical principles outlined in the Declaration of Helsinki. More details regarding each group recruited and examined are provided below.

\section{Healthy controls}

All these individuals have been clinically examined attesting an absence of gynaecological problems and interviewed personally for the study during their hospital outpatient visit. BCfree condition has been confirmed either by breast sonography or mammography or both imaging approaches. The examination reports used were not older than 6 months. Healthy controls demonstrated no history of any previously diagnosed breast pathology, no surgery performed due to breast lesions and no history of any severe gynaecologic disease including cancer other than breast malignancies or any systemic diseases such as diabetes mellitus, rheumatic diseases and neurological disorders.

\section{Breast cancer and FIA patients}

About $90 \%$ of these patients have been selected in the database and then contacted telephonically by an authorised person who have explained the meaning/rules of the study and interviewed the responders through the entire questionnaire. Ten per cent of these patients have been personally contacted and interviewed during their stay at the clinic. Imaging technologies have been applied for the first choice of the entire diagnostic procedure: digital mammography Hologic system, $2 \mathrm{D}+3 \mathrm{D}$ sonography-Voluson USG system E8 and E10 machine, Birads 0-6 classification scoring system with double reading of the radiologic approach. In case of positivity, the affected patients have undergone the biopsy analysis (either core needle or Mammotome's vacuum-assisted). Contextually, BC diagnosis has been confirmed by core needle biopsy prior to surgery or during surgery by frozen section in cases with non-palpable lesions, non-reachable lesions for biopsy prior to surgery and small lesions not indicated to core biopsy (stereotactic, core needle or Mammotome). The preoperative diagnosis of breast cancer was a part of the surgery and management planning. The diagnosis has been finalised by the molecular biological classification of the breast cancer subtype. Histopathological analysis (see below) has, further, 
allowed for distinguishing between BC malignancy and FIA benignancy.

\section{Histopathological analysis}

Tumour and lymph node specimens have been fixed in formalin and embedded in paraffin. Basic histological examination has been performed on 4-5- $\mu$ m-thin slides stained with haematoxylin and eosin. In selected cases, lymph nodes have been stained immunohistochemically to detect potentially disseminated tumour cells or local micrometastases in the tumour's surrounding area. Classical morphological indicators, such as type and histological grade, have been evaluated according to WHO criteria and Nottingham grading modification [20, 21]. The disease graduation and staging (tumour size and lymph node status) have been assessed according to the criteria of the latest TNM classification [22]. BC-specific biological parameters (oestrogen receptors (ER), progesterone receptors (PR) and HER-2 positive status) have been evaluated immunohistochemically; the result interpretation have been performed based on ASCO/CAP criteria released in the years 2010 and 2013 [23-25]. Tumours have been considered as being ER and PR positive, if at least $1 \%$ of neoplastic cells have stained positively. Her-2-positive tumours had to express $3+$ reaction in at least $10 \%$ of neoplastic cells. Cases with $2+$ reaction of Her-2 staining were considered as equivocal and have been analysed by fluorescent in situ hybridisation (FISH) to confirm or exclude Her-2 gene amplification. Conclusive positivity of Her-2 status in tumours has been defined as HER$2 / \mathrm{CEP} 17$ ratio $\geq 2.0$ or average HER-2 copy number $\geq 6.0$ signals per cell $[24,25]$. The molecular biological classification of breast cancer and consequent patient categorisation utilises the following system of the pathology-specific parameters:

$$
\begin{aligned}
& \text { a/ER + PR + HER2-Ki67low-luminal A } \\
& \text { b/ER+/-PR+/-HER2-Ki67high-luminal B } \\
& \text { c/ ER+/-PR+/-HER2+/Ki67 any-luminal C } \\
& \text { d/ER-PR-HER2 + Ki67high-Her2-positive } \\
& \text { e/ER-PR-HER2-Ki67high-Triple-negative breast } \\
& \text { cancer }
\end{aligned}
$$

\section{Statistical analysis}

For analytical and statistical evaluations, the data have been transferred to Microsoft Excel. SPSS Statistics v20.0.0 software (IBM, Armonk, New York, USA) has been applied. The prevalence of individual symptoms in groups of comparison has been evaluated and expressed in percentages. Pearson's chi-square test of associations has been applied. $P$ values equal or below 0.05 have been considered as statistically significant.

\section{Results}

\section{Age and menopausal status statistics}

Table 1 presents statistics provided for individual breast cancer subgroups and the group of comparison comprising breast cancer (BC)-free individuals. The latter demonstrates the parity in menopausal status. Amongst BC patients, the youngest is the "triple-negative" subgroup presenting $39 \%$ of premenopausal women. In contrast, the "luminal" and "Her2positive" subgroups both comprise over $90 \%$ postmenopausal women.

\section{Evaluation of the prevalence of individual FS symptoms}

In Fig. 1, results achieved are summarised demonstrating the prevalence of individual Flammer syndrome symptoms (115 ) in two main groups of comparison - "BC total" patients versus "BC-free" individuals as well as in individual subgroups of breast cancer. Higher prevalence in BC total (red) has been demonstrated for altogether 11 from 15 symptoms at the level of difference ranging between 5 and 19\%. The level of significance varies correspondingly with the most significant differences demonstrated for the symptoms "Pain" $(p=0.01)$, "Skin blotches" $(p=0.06)$, "Low blood pressure" $(p=0.11)$, and "Feeling cold" $(p=0.15)$.

Noteworthy, substantial differences have been recorded between individual BC subtypes, even in cases when the resulting $\mathrm{BC}$ total prevalence was non-significantly higher

\begin{tabular}{|c|c|c|c|c|}
\hline \multirow{2}{*}{$\begin{array}{l}\text { Menopausal } \\
\text { status }\end{array}$} & \multicolumn{3}{|l|}{ BC subtype } & \multirow{2}{*}{$\begin{array}{l}\text { BC-free, } \\
\text { patient } \\
\text { number }\end{array}$} \\
\hline & $\begin{array}{l}\text { LUMINAL, } \\
\text { patient } \\
\text { number }\end{array}$ & $\begin{array}{l}\text { HER2, } \\
\text { patient } \\
\text { number }\end{array}$ & $\begin{array}{l}\mathrm{TN}, \\
\text { patient } \\
\text { number }\end{array}$ & \\
\hline Premenopausal & 5 & 2 & 7 & 38 \\
\hline Postmenopausal & 46 & 11 & 11 & 35 \\
\hline $\begin{array}{l}\text { Patients' age } \\
\text { mean } \\
\text { (min-max) } \\
\text { years }\end{array}$ & $\begin{array}{l}61.84 \\
\quad(36-85)\end{array}$ & $\begin{array}{l}61.85 \\
\quad(46-85)\end{array}$ & $\begin{array}{l}54.11 \\
\quad(33-77)\end{array}$ & $\begin{array}{l}50.19 \\
\quad(19-89)\end{array}$ \\
\hline
\end{tabular}
compared to BC-free. Hence, in case of the symptom 2

Table 1 Age and menopausal status statistics; the data are provided for individual breast cancer subgroups (LUMINAL, HER2-positive and $\mathrm{TN}$ - triple-negative) and the group comprising breast cancer-free individuals; age mean differences are statistically non-significant 
Fig. 1 Evaluation of the prevalence of individual symptoms (1-15) of the "Flammer syndrome" phenotype in two groups of comparison: "breast cancer-diseased" (BC total) versus "breast cancer-free" (BCfree). Therein, the entire breast cancer patient pool ("BC total") has been additionally analysed in subgroups stratified according to the individual BC subtypes as "LUMINAL", "HER2" positive and TN (triple negative) breast cancer. For more details regarding the patient stratification, see the "Materials and methods" section. The prevalence in each individual group is presented by percentage of individuals who have responded to the corresponding question with "frequently" and "sometimes" pooled together. Responders answering with "I do not know" have been excluded from the overall numbers/calculations. Question-specific notes: Question 6- the ratio between "I do not feel thirsty and drink little" and "I feel much thirsty and drink a lot" has been calculated and expressed in X times; Question 12-answers "very slim" and "slim" have been pooled together and presented in \%

("Feeling cold"), the difference between both main groups comprises 10\% ( $p=0.159)$; however, the prevalence in luminal $\mathrm{BC}$ is two times compared with Her-2.

The BC-free (green) group demonstrates higher prevalence only for one symptom (8. "Accompanying symptoms"); further three symptoms (11. "Smell perception", 12. "Low body weight in early adulthood", and 13. "Perfectionism") demonstrate similar prevalence in both groups of comparison. However, in case of these four $(8,11,12,13)$ symptoms, remarkable differences have been demonstrated for the prevalence, when individual BC subgroups have been compared against each other. There are two most representative examples for that, namely,

1. Although symptom 8 ("Accompanying symptoms") demonstrates slightly lower (8\%) prevalence in $\mathrm{BC}$ total, Her2-postive BC subtypes suffer from this symptom more frequently than do $\mathrm{BC}$ free and demonstrates two times higher prevalence compared to the Luminal $\mathrm{BC}$ subtype.

2. Symptom 12 ("Low body weight in early adulthood") is in average equal for both main groups of comparison. However, across the panel, while luminal BC subtype demonstrates the lowest prevalence by $47 \%$, obviously low body weight in early adulthood is highly prevalent in both Her2-positive and triple-negative $\mathrm{BC}$ subtypes by 69 and $72 \%$, respectively.

Concluding Table 2 shows four basic clusters of symptoms, where

- Cluster A (marked in green colour) represents the group of symptoms with higher prevalence in $\mathrm{BC}$ in general as well as all individual subgroups.

- Cluster B (marked in blue colour) represents the group of symptoms with higher prevalence in $\mathrm{BC}$, in general, but differential prevalence in individual BC subgroups.
1.Do you suffer from cold hands and/or feet?

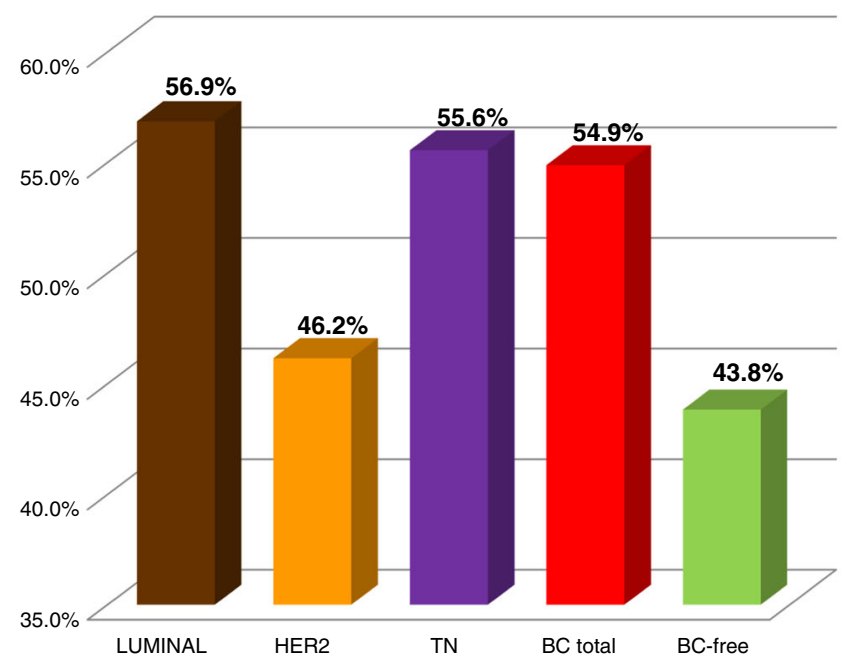

2. Do you feel cold when you sit down quietly for some time or when you are not moving?

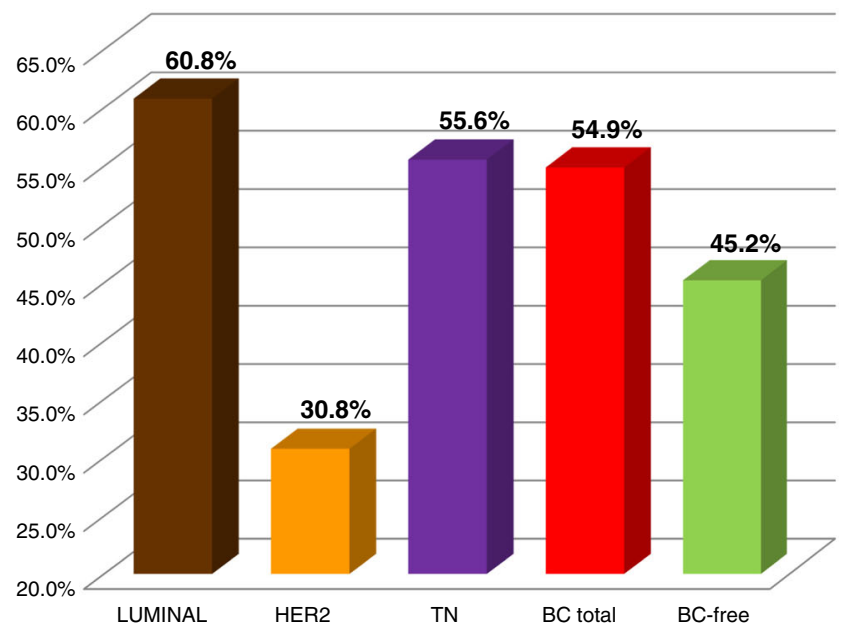

3. Do you have (have you had earlier) a low blood pressure?

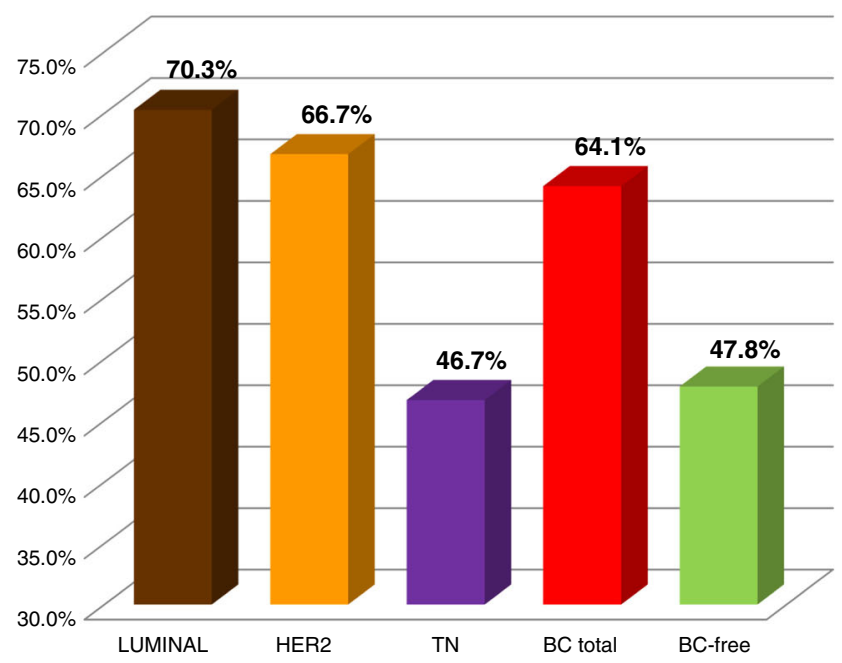


4. Do you ever feel dizzy when you suddenly stand up from a lying (or resting) position?

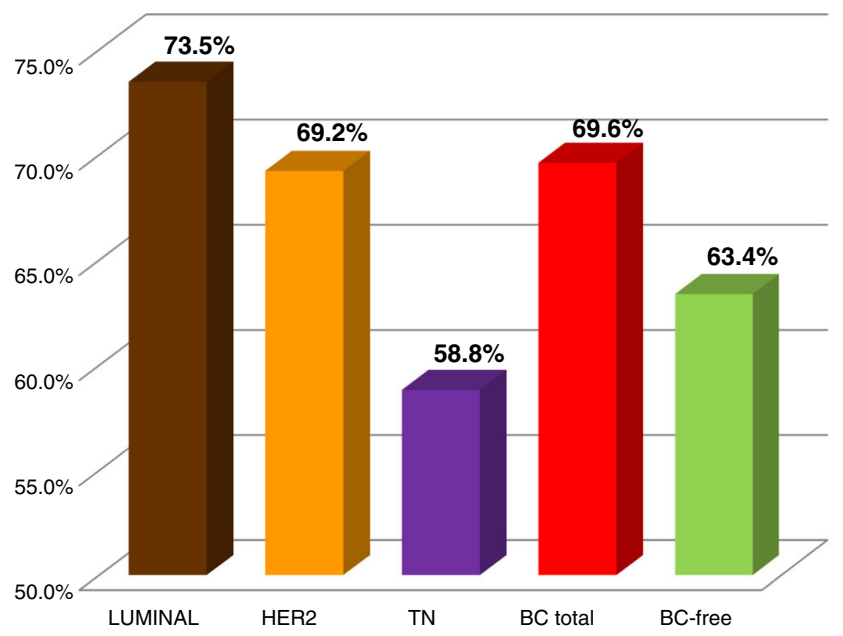

5. Do you need a relatively long time to fall a sleep?
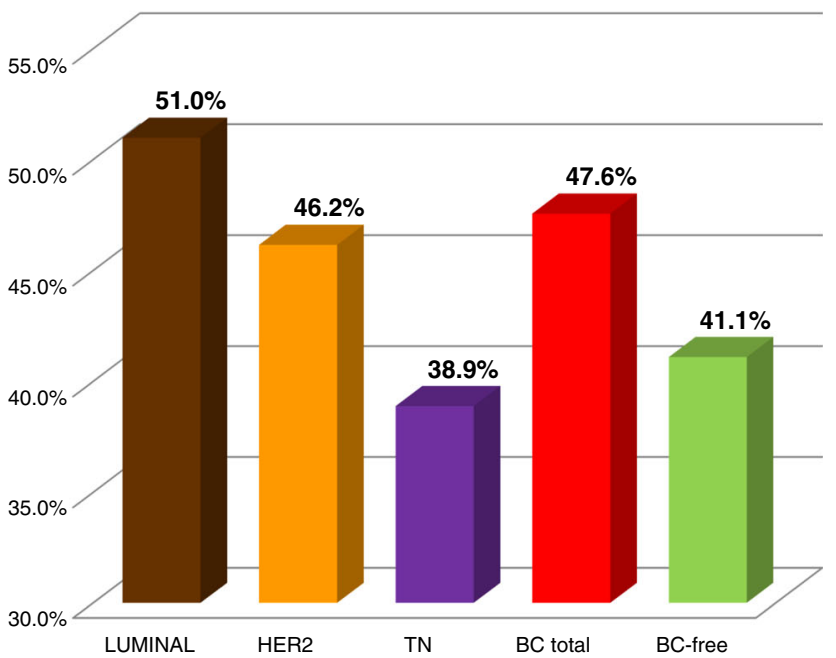

6. How is your feeling of thirst?

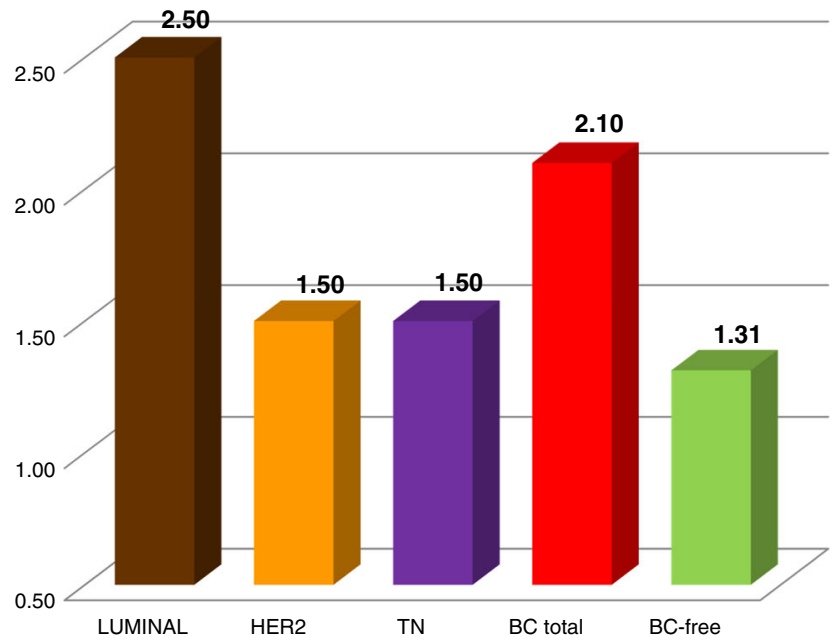

Fig. 1 (continued)

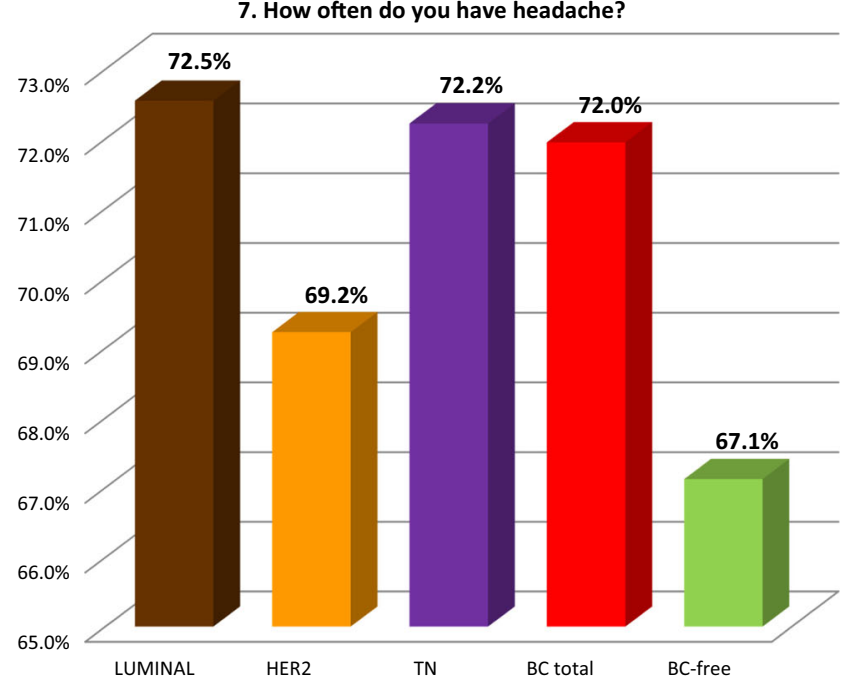

8. In case you suffer from migraines, do you have accompanying symptoms (e.g. visual disturbances, transient altered sensation (e.g. cribbling) in your arms or in your legs etc.)?

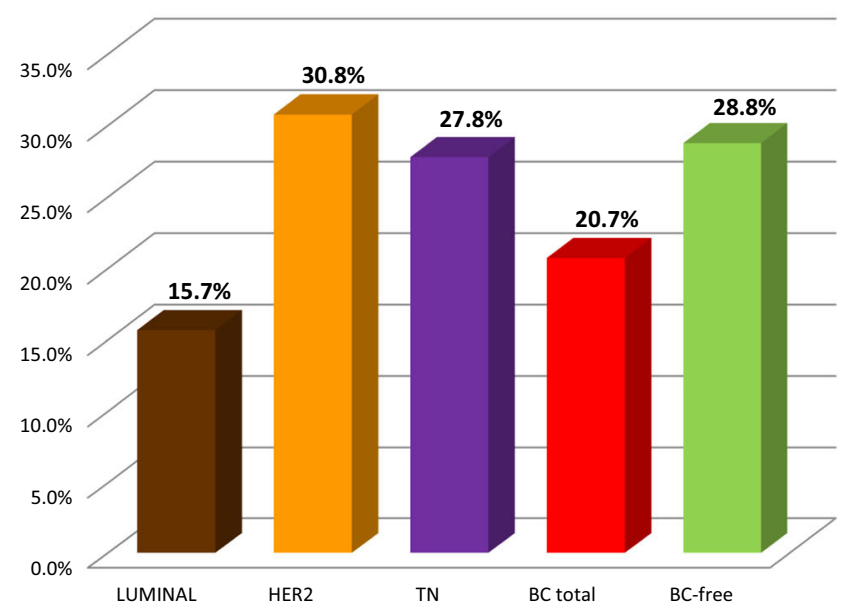

9. If you have to take medications (other than painkillers), do you have the feeling that you react strongly to them and/or that you would feel better, if you would take a lower dosis than that which is normally prescribed?

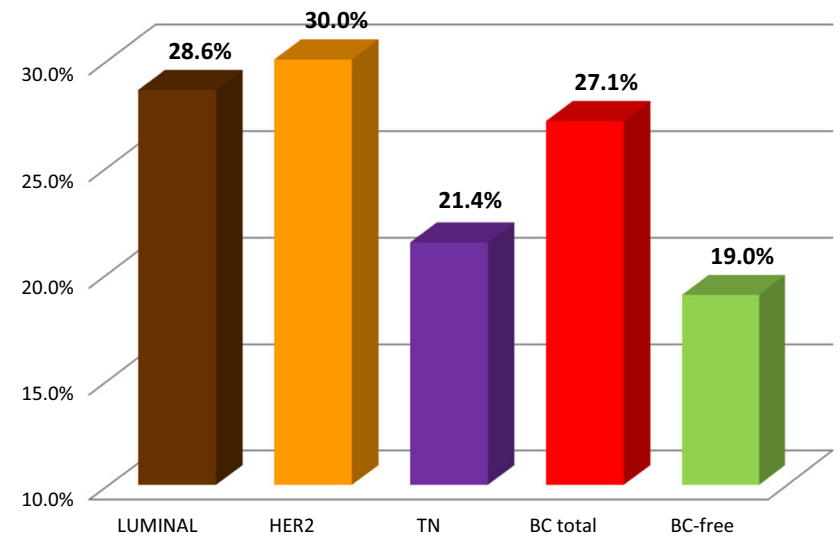

Fig. 1 (continued) 
10. Do you suffer from any type of pain which you would have to take pain killers for?

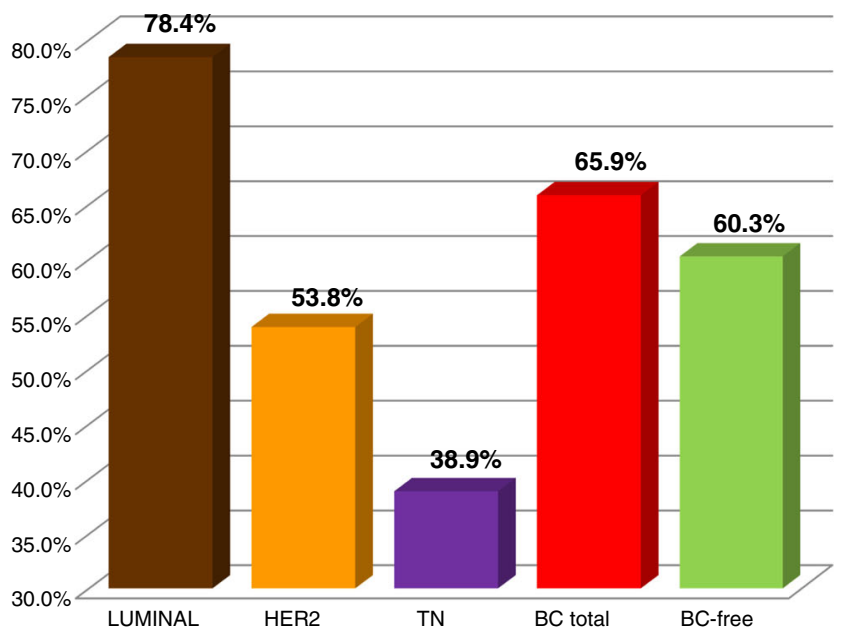

11. How well can you smell: Can you smell things that other people don't smell or that others smell to a lesser extent?

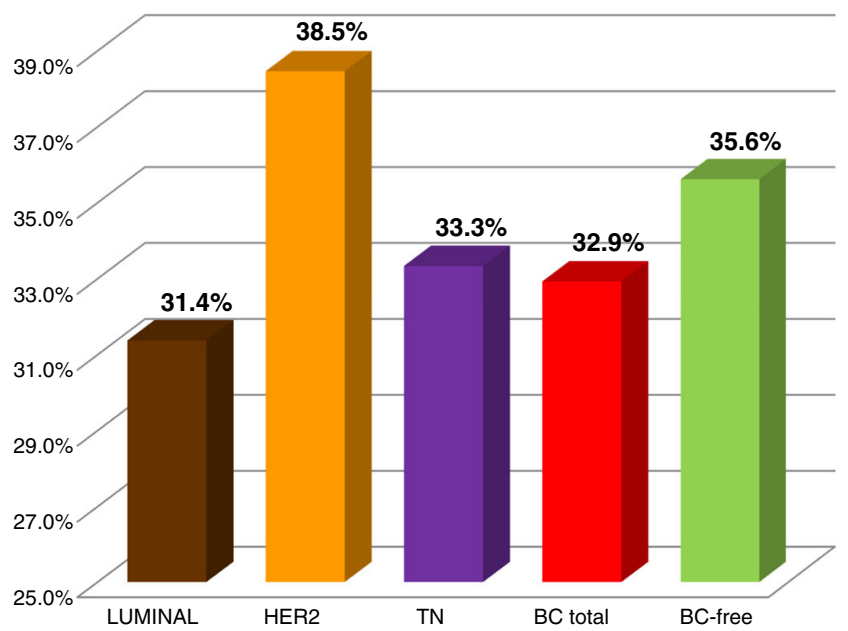

12. Were you slim at $\mathbf{2 0 - 3 0}$ years of age?

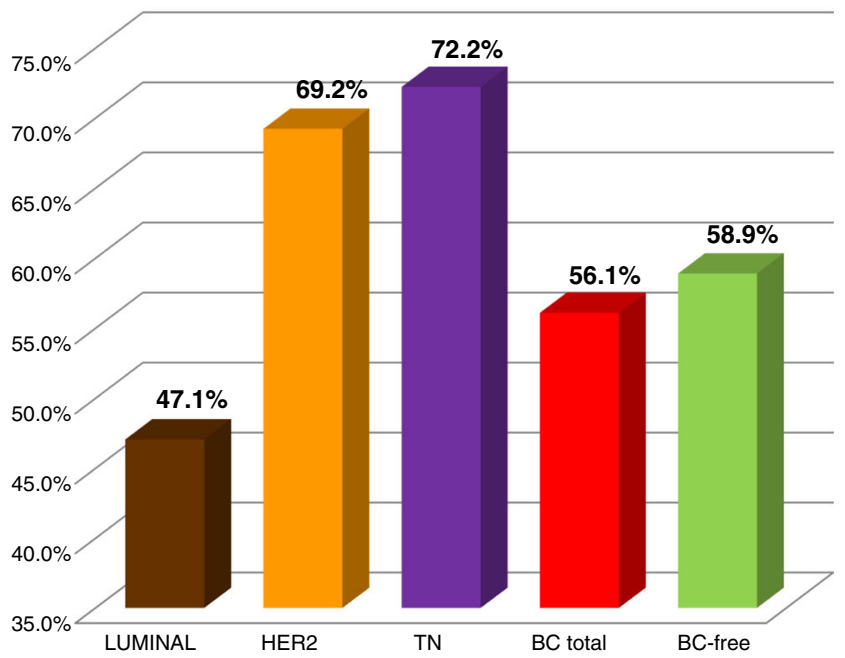

Fig. 1 (continued)
13. If you have to judge yourself (e.g. in your work), would you say that you are particularly reliable with a tendency towards perfectionism?

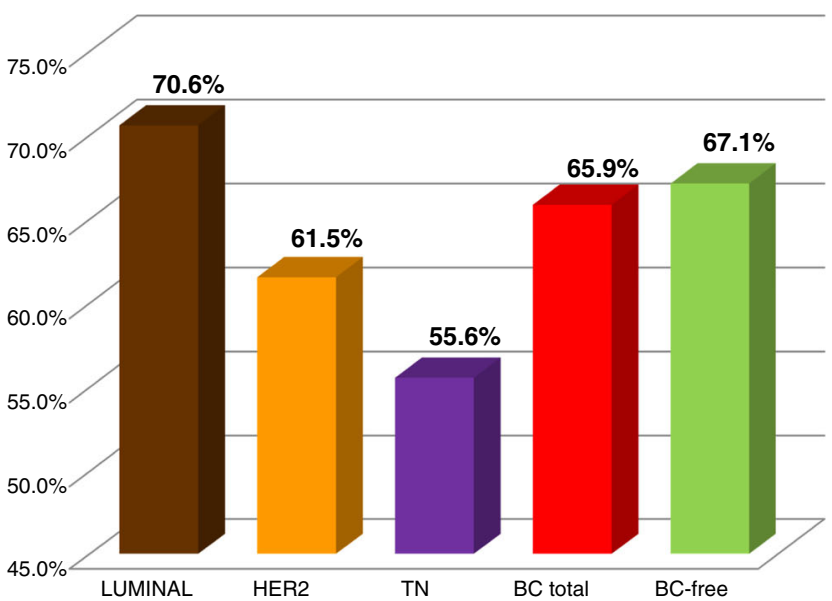

14. Have you had phases in your life in which you had ringing in your ears (tinnitus)?

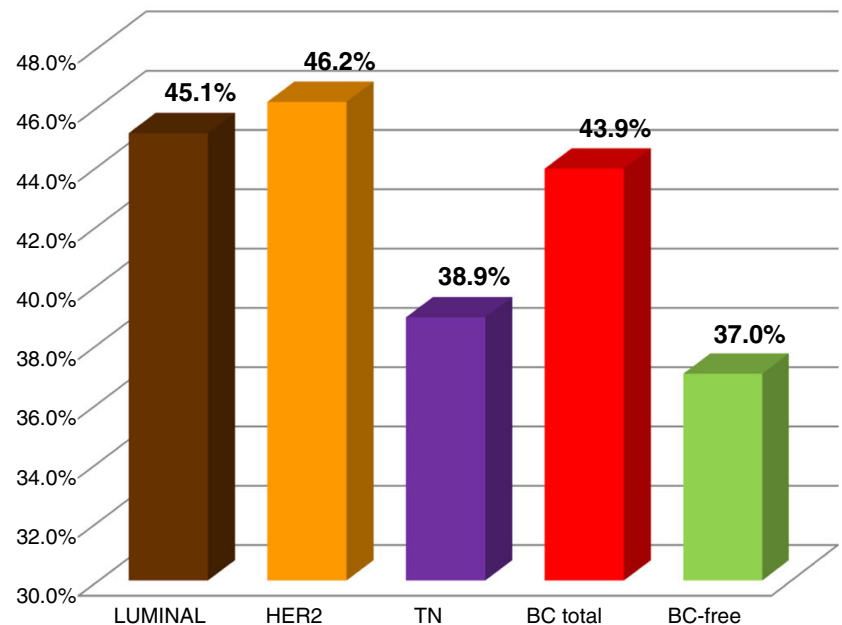

15. Have you noticed reversible blotches (white or red) on your skin when you were very excited or angry (e.g. in stress)?

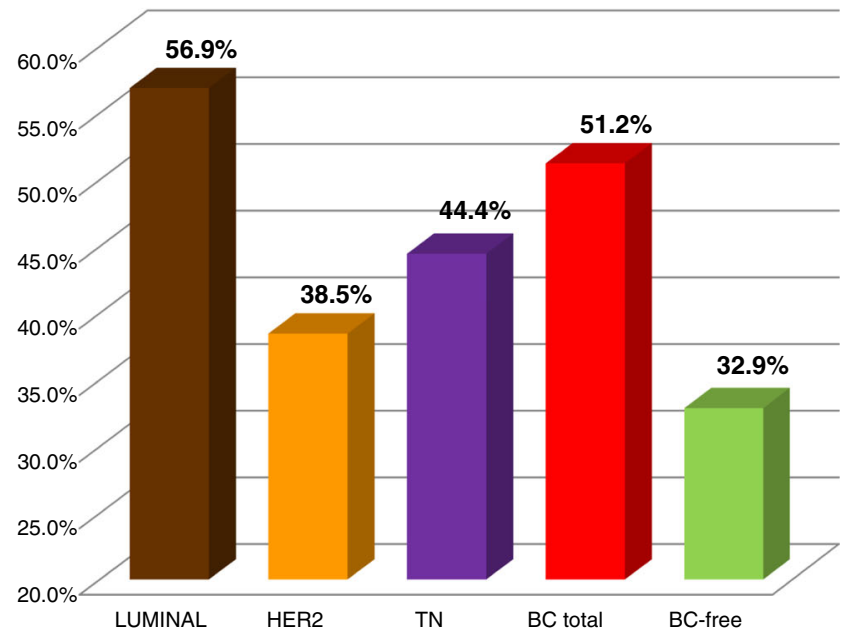

Fig. 1 (continued) 
Table 2. Symptoms of the "Flammer syndrome" recorded in breast cancer-diseased versus breast cancer-free groups of comparison; the table summarises the results demonstrated in Fig. 1 by utilising the following system: with "+", the group is noted which demonstrates higher prevalence of the corresponding symptom (above the lowest average of the groups of comparison); with "--", the group is noted which demonstrates lower prevalence of the corresponding symptom (lowest average and below it); "=" means both groups of comparison demonstrate similar prevalence of the corresponding symptom; "++" means values sufficiently over the highest average; "-" means values sufficiently below the highest average; ellipses mark the symptoms which are more prevalent in individual subgroups; $\sum$ criteria summarise the number of symptoms which are more (+) or less (-) prevalent in corresponding sub/groups

\begin{tabular}{|c|c|c|c|c|c|c|}
\hline \multirow[t]{2}{*}{ Nr. } & \multirow[t]{2}{*}{ Symptoms } & \multicolumn{4}{|c|}{ Breast cancer diseased } & \multirow{2}{*}{$\begin{array}{c}\text { Breast } \\
\text { cancer } \\
\text { free }\end{array}$} \\
\hline & & Total & Luminal & Her2 & TN & \\
\hline 1 & Cold hands & + & + & + & + & - \\
\hline 6 & Thirst & + & ++ & + & + & - \\
\hline 7 & Headache & + & + & + & + & - \\
\hline 9 & Drug sensitivity & + & + & + & + & - \\
\hline 14 & Tinnitus & + & + & + & + & - \\
\hline 15 & Skin blotches & + & ++ & + & ++ & - \\
\hline 2 & Feeling cold & + & ++ & - & + & - \\
\hline 3 & $\begin{array}{l}\text { Low blood } \\
\text { pressure }\end{array}$ & + & ++ & ++ & $=$ & - \\
\hline 4 & Dizziness & + & + & + & - & - \\
\hline 5 & Sleep onset & + & + & + & $=$ & - \\
\hline 10 & Pain & + & + & - & -- & - \\
\hline 11 & Smell perception & $=$ & - & + & $=$ & $=$ \\
\hline 12 & $\begin{array}{l}\text { Low body weight } \\
\text { in early adulthood }\end{array}$ & $=$ & -- & $<++$ & ++ & $=$ \\
\hline 13 & Perfectionism & $=$ & + & - & - & $=$ \\
\hline 8 & $\begin{array}{l}\text { Accompanying } \\
\text { symptoms }\end{array}$ & - & -- & 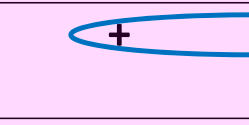 & \pm & + \\
\hline & $\sum$ criteria & $11+$ & $\begin{array}{c}12+ \\
(8+\& 4++)\end{array}$ & $\begin{array}{c}12+ \\
(10+\& 2++)\end{array}$ & $\begin{array}{c}9+ \\
(7+\& 2++)\end{array}$ & $1+$ \\
\hline & & $\begin{array}{l}3= \\
1-\end{array}$ & $\begin{array}{l}2-- \\
1-\end{array}$ & 3- & $\begin{array}{l}3= \\
1-- \\
2-\end{array}$ & $\begin{array}{l}3= \\
11-\end{array}$ \\
\hline
\end{tabular}

- Cluster C (marked in yellow colour) represents the group of symptoms with the prevalence which, in general, is similar for both, $\mathrm{BC}$ diseased and $\mathrm{BC}$-free individuals, but varies between individual $\mathrm{BC}$ subgroups. 
- Cluster D (marked in red colour) represents a symptom with the prevalence which, in general, is lower for BC-diseased patients, however, varies between individual $\mathrm{BC}$ subgroups.

Cluster A represents a consolidate group of symptoms relevant for all BC patients investigated in this study. In contrast, clusters B, C and D demonstrate high level of plurality regarding their prevalence in $\mathrm{BC}$ patients investigated.

\section{Discussion}

Here, we present highly innovative concepts considering investigation of specific syndromes and symptoms underestimated till now in relationship with $\mathrm{BC}$ predisposition and development. Consequently, the purpose of our pilot project was to evaluate potential trends in prevalence of the "Flammer syndrome" (FS) in breast cancer (BC) patient cohort as justified above. For this very first pilot project performed in the field of FS/BC, relatively modest patient numbers have been recruited, which, however, are big enough to estimate potential relevance of FS for BC. A great advantage of the study design is that all participating patients and individuals have been thoroughly examined utilising conventional methodology of medical imaging and laboratory medicine and, consequently, selected for the study strictly according to the including and excluding criteria chosen, namely

- breast cancer-free health condition

- breast cancer malignancies stratified according to the globally accepted classification

- no history of any severe gynaecologic disease including cancer other than breast malignancies

- no history of any systemic disease such as diabetes mellitus, rheumatic diseases and neurological disorders.

Since our current project is a pioneer one in the field of FS/ $\mathrm{BC}$, the study has been performed in a hypothesis-free way regarding potential influence of the specific biological parameters such as age, menopausal status and breast cancer-related risk factors (such as a genetic component, smoking, inappropriate alcohol consumption, breast feeding, prolonged menarche, etc.) that represents the main limitation of the current study and requests much higher numbers of the study participants for statistically relevant patient collectives of comparison. In our pilot study, the resulting groups recruited reflect usual profiles of the breast cancer patient cohorts, namely the prevalence of the luminal subtype is significantly higher in $\mathrm{BC}$ total compared to both Her2-positive and triple-negative BC; the luminal subgroup is older and about $90 \%$ of the patients in luminal and Her2-positive subgroups are postmenopausal in contrast to the triple-negative $\mathrm{BC}$ subgroup comprising about $40 \%$ of premenopausal women (see Table 1). Potential influence of those unmatched parameters on FS prevalence in individual $\mathrm{BC}$ subtypes should be investigated additionally by the goaldedicated projects. Consequently, the main goal of our pilot study was to verify the main hypothesis proposing the relevance of FS for BC appearance and to estimate potential trends in correlation individual FS symptoms with BC pathology.

The results achieved here support the main hypothesis of the project clearly demonstrating the tendency of $\mathrm{BC}$ patients to an increased prevalence of FS symptoms compared to the disease-free individuals. As summarised in Table 2, the prevalence of 11 symptoms is higher in breast cancer patients, while only one symptom (8. "Accompanying symptoms") was demonstrated to be more prevalent in BC-free individuals. Furthermore, even in this case as well as in further three cases of the neutral prevalence (11. "Smell perception", 12. "Low body weight in early adulthood" and 13 . "Perfectionism"), remarkable differences have been demonstrated, when individual BC subgroups have been compared against each other. This actuality emphasises an extremely strong heterogeneity of the patients within the entire $\mathrm{BC}$ cohort. Obviously, FS prevalence does not create any exception regarding $\mathrm{BC}$ heterogeneity and therefore motivates followup studies investigating statistically significant correlations between single FS symptoms and specific BC patient profiles. To this end, cluster A (Table 2) as the best consolidate group of symptoms relevant for all $\mathrm{BC}$ patients investigated in this study, is expected to be more close to its potential application in daily medical practice by primary care units and family doctors and innovative screening programmes. In contrast, more research efforts are still needed to interpret the symptoms' plurality in clusters B, C and D that might lead to novel approaches in $\mathrm{BC}$ patient stratification and more clear discrimination between individual $\mathrm{BC}$ subtypes.

\section{What are the main "take-home messages" of this study?}

\section{Concluding remarks and outlook}

\section{Breast cancer is a systemic disease}

All individual parameters (1-15), the prevalence of which has been evaluated in the study, are functionally well linked to each other and altogether demonstrate systemic alterations of the affected person. Although individual $\mathrm{BC}$ subtypes differ from each other in the prevalence distribution, all of them demonstrate systemic alterations compared to the diseasefree individuals. 
Crucial role of the cardiovascular component in the $B C$ pathology

The great advantage of the evaluation system utilising the FSquestionnaire is that several symptoms are used as a conditionspecific biomarker panel being functionally linked to each other and, therefore, eliminate any contingency usually handicapping single biomarkers. Hence, symptoms 1 ("Cold extremities"), 2 ("Feeling cold"), 3 ("Low blood pressure"), 4 ("Dizziness"), 8 ("Accompanying symptoms") and 15 ("Skin blotches appearing under stress conditions") create highly specific biomarker panel demonstrating great synergies in a complex evaluation of impacts by the cardiovascular component. All BC subgroups responded to this panel, although differing by subgroup-specific accents. For instance, whereas luminal BC patients have been recorded as the strongest responders towards "Feeling cold" and "Low blood pressure", Her-2-positive patients responded strongly towards "Low blood pressure" but mildly towards "Feeling cold". The meaning of those subgroup-specific differences should be further investigated.

\section{Local and systemic hypoxic effects in BC and metastatic disease}

Strong impacts by the above discussed cardiovascular biomarker panel lead to a conclusion about local and systemic hypoxic effects to be expected in $\mathrm{BC}$ patients responding to this panel of FS symptoms. The role of these effects in the BC pathology and related metastatic disease emerges deep investigations.

\section{Interpretation of headache, migraine and dizziness in $B C$}

"Headache" and "migraine" are frequently discussed for breast cancer patient cohorts with highly controversial study outcomes and conclusions. The reason for that might be a missing context, which should obligatory include specific biomarker panels by FS symptoms, such as cardiovascular component (see above), altered regulation of pain and thirst (see below).

\section{Altered sense regulation in $B C$ aetiology?}

Our study makes it obvious that BC patients may suffer from altered systems of the sense regulation and relevant receptors, due to their response to the biomarker panel comprising the following symptoms: 6 ("Thirst regulation"), 7 ("Headache"), 10 ("Pain") and 11 ("Smell perception"). Functional links between these symptoms make a good sense for potential interpretations. Hence, underrepresented feeling of thirst may result in strong body dehydration and disturbed detoxification pathways, which in turn may cause significant toxic effects increasing $\mathrm{BC}$ risks as discussed in the recently published article "Feeling cold and other underestimated symptoms in breast cancer: anecdotes or individual profiles for advanced patient stratification?" [2]. Body dehydration and insufficient detoxification may synergistically lead to increased frequency of headache which is a very useful indicator of impaired systemic regulations. Contextually, pain (e.g. headache) plays a crucial role indicating systemically impaired processes. To this end, symptom 10 ("Pain") provides very important information recording, on one side, the highest responder rates in the luminal (78.4\%), and on the other side, the lowest rates in the triplenegative BC (38.9\%). This is a very strong indication to investigate pain regulation specifically in the triplenegative $\mathrm{BC}$ patients who might be potentially pain resistant and, therefore, inadequately reacting towards corresponding stimuli.

\section{Drug sensitivity may be potentially altered in $B C$}

Symptom 9 has recorded the highest responder rates in the Her-2-positive BC. This may potentially indicate altered drug sensitivity in BC patients suffering from FS. Altered drug sensitivity has been demonstrated for FS individuals and glaucoma patients with FS $[3,7,26]$.

\section{Thermoregulation symptoms in BC pathology}

The symptom 1 ("Cold extremities") is clearly linked to the disturbed microcirculation and consequently to the local and systemic hypoxic effects discussed above. However, the meaning of "Feeling cold" (symptom 2) may have different interpretations. Therefore, the prevalence of both symptoms is not obligatory correlating completely in the same groups of comparison as it has been demonstrated in our study. Indeed, $\mathrm{BC}$ patients complain more frequently about deficits in achieving thermal comfort: they feel excessively hot or cold even in situations when disease-free attendees are well comfortable with ambient temperature conditions [27]. "Feeling inadequately cold" may be interpreted in two ways. First, the heat production is not effective enough to maintain the physiologic body temperature, and the systemic mitochondrial dysfunction and DNA damage/misguided repair have been proposed as implemented in the BC pathology [28, 29]. The second interpretation is the fever or fever-like conditions with excessive chill attacks regulated by proinflammatory cytokines, the expression profiles of which are frequently altered in cancer patients [30]. More detailed discussion on the matter is presented in the recently published article [2].

\section{Body shape impacts $B C$}

Evaluation of the symptom 12 has revealed the strong subgroup specificity in BC regarding body weight in early adulthood: while luminal BC subtype demonstrates the lowest prevalence by $47 \%$, obviously low body weight in early 
adulthood is highly prevalent in both Her2-positive and triplenegative BC subtypes by $69 \%$ and $72 \%$, respectively. This actuality creates questions regarding body shape impacts in $\mathrm{BC}$ pathology as discussed earlier [1].

\section{The role of psychological factors in BC pathology}

Psychological factors play a role in $\mathrm{BC}$ predisposition, progression and individual outcomes. The biomarker panel comprising symptoms 5 ("Sleep onset"), 11 ("Smell perception") and 13 ("Perfectionism") strongly promote this concept and motivate additional studies estimating the impacts of psychological factors and concomitantly regulated patho/ physiological process in $\mathrm{BC}$ pathology.

Acknowledgements The authors thank Prof. Dr. Josef Flammer, University of Basel, Switzerland for his valuable recommendations during the preparation and performance of the actual multicentred project. Further, the authors thank Dr. Erika Halasova for her valuable help in organising the scientific collaboration between the EPMA and Jessenius Faculty of Medicine, Martin, Slovak Republic.

Authors' contributions Olga Golubnitschaja is the project coordinator who has created the main scientific ideas and hypotheses presented in the manuscript; she has drafted the manuscript. Pavol Zubor has coordinated the research, patient recruitment and data analysis performed in Slovakia and supervised Alexandra Gondova and Petra Kasajova. Pavol Zubor, Alexandra Gondova and Petra Kasajova have analysed the patient database and selected the patients for the study. Alexandra Gondova and Petra Kasajova interviewed the patients and collected the data. Jiri Polivka Jr. has performed statistical analysis. Jan Danko has contributed to the project by the expertise in breast cancer research and treatment. Katarzyna Konieczka has contributed by the expertise in "Flammer syndrome". All the authors have read and approved the final manuscript.

Compliance with ethical standards All procedures performed in the current study involving human participants were in accordance with the ethical standards of the institutional and/or national research committee and with the 1964 Helsinki Declaration and its later amendments or comparable ethical standards.

Funding This multicentred study has been supported by the following organisations:

- European Association for Predictive, Preventive and Personalised Medicine, EPMA, Brussels, Belgium

- Schwickert-Foundation, Switzerland

- Slovak Research and Development Agency (under the contract no. APVV-14-0815), the Scientific Grant Agency, Ministry of Education, Science and Research, Slovak Republic

- National Sustainability Program I (NPU I) Nr. LO1503, Ministry of Education Youth and Sports, Czech Republic and MH CZ-DRO (Faculty Hospital in Plzen-FNP1, 00669806).

Competing interests The authors declare that they have no competing interests.

\section{References}

1. Golubnitschaja O, Debald M, Yeghiazaryan K, Kuhn W, Pešta M, Costigliola V, Grech G. Breast cancer epidemic in the early 21st century: evaluation of risk factors, cumulative questionnaires and recommendations for preventive measures. Tumor Biol. 2016;37(10):12941-57. doi:10.1007/s13277-016-5168-x.

2. Golubnitschaja O. Feeling cold and other underestimated symptoms in breast cancer: anecdotes or individual profiles for advanced patient stratification? EPMA J. 2017;8(1):17-22. doi:10.1007/ s13167-017-0086-6.

3. Konieczka K, Ritch R, Traverso CE, Kim DM, Kook MS, Gallino A, et al. Flammer syndrome. EPMA J. 2014;5(1):11. doi:10.1186/ 1878-5085-5-11.

4. Yeghiazaryan K, Flammer J, Orgül S, Wunderlich K, Golubnitschaja O. Vasospastic individuals demonstrate significant similarity to glaucoma patients as revealed by gene expression profiling in circulating leukocytes. Mol Vis. 2009;15:2339-48.

5. Flammer J, Konieczka K, Flammer AJ. The primary vascular dysregulation syndrome: implications for eye diseases. EPMA J. 2013;4(1):14. doi:10.1186/1878-5085-4-14.

6. Yeghiazaryan K, Flammer J, Golubnitschaja O. Predictive molecular profiling in blood of healthy vasospastic individuals: clue to targeted prevention as personalised medicine to effective costs. EPMA J. 2010;1(2):263-72. doi:10.1007/s13167-010-0032-3.

7. Golubnitschaja O, Yeghiazaryan K, Flammer J. Key molecular pathways affected by glaucoma pathology: is predictive diagnosis possible? EPMA J. 2010;1(2):237-44. doi:10.1007/s13167-010-0031-4.

8. Golubnitschaja O, Yeghiazaryan K, Costigliola V, Trog D, Braun M, Debald M, et al. Risk assessment, disease prevention and personalised treatments in breast cancer: is clinically qualified integrative approach in the horizon? EPMA J. 2013;4(1):6. doi:10. 1186/1878-5085-4-6.

9. Flammer J, Konieczka K. Retinal venous pressure: the role of endothelin. EPMA J. 2015;6:21. doi:10.1186/s13167-015-0043-1.

10. Fang L, Baertschi M, Mozaffarieh M. The effect of flammersyndrome on retinal venous pressure. BMC Ophthalmol. 2014;14:121. doi:10.1186/1471-2415-14-121.

11. Fraenk1 SA, Mozaffarieh M, Flammer J. Retinal vein occlusions: the potential impact of a dysregulation of the retinal veins. EPMA J. 2010;1(2):253-61. doi:10.1007/s13167-010-0025-2.

12. Konieczka K, Flammer AJ, Todorova M, Meyer P, Flammer J. Retinitis pigmentosa and ocular blood flow. EPMA J. 2012;3(1): 17. doi:10.1186/1878-5085-3-17.

13. Konieczka K, Koch S, Binggeli T, Schoetzau A, Kesselring J. Multiple sclerosis and primary vascular dysregulation (Flammer syndrome). EPMA J. 2016;7:13. doi:10.1186/s13167-016-0062-6.

14. Konieczka K, Fränkl S. Primäre vaskuläre dysregulation und Glaukom (primary vascular dysregulation and glaucoma). Z Prakt Augenheilkd. 2013;34:207-15.

15. Konieczka K. Flammer syndrome, in: Brähler E, Hoefert H-W. Lexikon der Modernen Krankheiten. 2014, Medizinisch Wissenschaftliche Verlagsgesellschaft. Berlin. Deutschland.

16. Konieczka K, Choi HJ, Koch S, Schoetzau A, Küenzi D, Kim DM. Frequency of symptoms and signs of primary vascular dysregulation in Swiss and Korean populations. Klin Monatsbl Augenheilkd. 2014;231:344-7.

17. Konieczka K, Koch S, Schoetzau A, Todorova MG. Increased prevalence of Flammer syndrome in patients with retinitis pigmentosa. Klin Monatsbl Augenheilkd. 2016;233(4):448-52. doi:10.1055/s0041-111802.

18. Konieczka K, Todorova MG, Chackathayil TN, Henrich PB. Cilioretinal artery occlusion in a young patient with Flammer syndrome and increased retinal venous pressure. Klin Monatsbl Augenheilkd. 2015;232:576-8. doi:10.1055/s-0035-1545774. 
19. Konieczka K, Flammer J, Sternbuch J, Binggeli T, Fraenkl S. Leber's hereditary optic neuropathy, normal tension glaucoma, and Flammer syndrome: long term follow-up of a patient. Klin Monatsbl Augenheilkd. 2017; doi:10.1055/s-0042-119564.

20. Lakhani SR, Ellis IO, Schnitt SJ, Tan PH, van de Vijver MJ. WHO classification of tumours of the breast. 4th ed. Lyon: IARC; 2012.

21. Elston CW, Ellis IO. Pathological prognostic factors in breast cancer. I. The value of histological grade in breast cancer: experience from a large study with long-term follow-up. Histopathology. 1991;19(5):403-10.

22. Edge SB, Compton CC. The American joint committee on cancer: the American joint committee on cancer: the 7th edition of the AJCC cancer staging manual and the future of TNM. Ann Surg Oncol. 2010;17(6):1471-4. doi:10.1245/s10434-010-0985-4.

23. Hammond MEH, Haves DF, Wolff AC, Mangu PB, Temin S. American Society of Clinical Oncology/College of American Pathologists guideline recommendations for immunohistochemical testing of estrogen and progesterone receptors in breast cancer. Arch Pathol Lab Med. 2010;134:E1-E16.

24. Wolff AC, Hammond ME, Hicks DG, Dowsett M, McShane LM, Allison KH, Allred DC, Bartlett JM, Hayes DF, et al. American Society of Clinical Oncology; College of American Pathologists. Recommendations for human epidermal growth factor receptor 2 testing in breast cancer: American Society of Clinical Oncology/ College of American Pathologists clinical practice guideline update.
J Clin Oncol. 2013;31(31):3997-4013. doi:10.1200/JCO.2013.50. 9984.

25. Wolff AC, Hammond ME, Hicks DG, Dowsett M, McShane LM, Allison KH, Allred DC, Bartlett JM, et al. Recommendations for human epidermal growth factor receptor 2 testing in breast cancer: American Society of Clinical Oncology/College of American Pathologists clinical practice guideline update. Arch Pathol Lab Med. 2014;138(2):241-56. doi:10.5858/arpa.2013-0953-SA.

26. Golubnitschaja O, Flammer J. What are the biomarkers in glaucoma? Surv Ophthalmol. 2007;52(Suppl 2):S155-61. doi:10.1016/j. survophthal.2007.08.011.

27. Kokolus K, Hing CC, Repasky EA. Feeling too hot or cold after breast cancer: is it just a nuisance or a potentially important prognostic factor? Int J Hyperth. 2010;26(7):662-80. doi:10.3109/ 02656736.2010 .507235 .

28. Mencalha A, Victorino VJ, Cecchini R, Panis C. Mapping oxidative changes in breast cancer: understanding the basic to reach the clinics. Anticancer Res. 2014;34(3):1127-40.

29. Liu X, Feng D, Liu D, Wang S, Yu X, Dai E, Wang J, Wang L, Jiang W. Dissecting the origin of breast cancer subtype stem cell and the potential mechanism of malignant transformation. PLoS One. 2016;11(10):e0165001. doi:10.1371/journal.pone.0165001.

30. Kurzrock R. The role of cytokines in cancer-related fatigue. Cancer. 2001;92(S6):1684-8. 